Methodological aspects and updates of computational models

\title{
Case study on the improved use of collective dose for nuclear and/or radiological emergencies
}

\author{
J. CAMPS, G. OLYSLAEGERS, D. BRAEKERS
}

\begin{abstract}
The improved use of collective dose following the 2007 ICRP recommendations is studied for nuclear and radiological emergency situations. To study the accumulation of low individual doses to the collective dose the collective dose is calculated as a function of the minimum individual dose for three cases including nuclear as well as radiological accidents. It is found that the use of collective dose in this way can have an added value in nuclear emergency preparedness and response in particular related to decisions on overall protective actions following an accident.
\end{abstract}

Keywords: Nuclear and radiological emergency/collective dose/ICRP recommendations

\section{Introduction}

The 2007 Recommendations of the International Commission on Radiological Protection (ICRP) emphasizes that the collective (effective) dose should only be used and understood as an instrument for the optimization of radiological protection and comparing radiological technologies (ICRP, 2007). It should be avoided to use the collective dose quantity for the computation of cancer deaths involving trivial exposures over extended periods to large populations because of the large uncertainties related to e.g. the risk coefficients in the very low dose range.

Related to nuclear and radiological emergency exposure situations, the ICRP 2007 recommendations underline the importance of justifying and optimizing the overall protection strategy. On the other hand nuclear and/or radiological emergencies can give individual exposures over a large dose range, for long periods and extended geographical regions. The use of collective dose related to emergency exposure for optimizing radiological protection is consequently a challenging issue and not frequently used in nuclear emergency response and preparedness.

SCK $\cdot \mathrm{CEN}$, Belgian Nuclear Research Centre, Institute for Environment, Health and Safety, Boeretang 200, B-2400 Mol, Belgium. 
However, collective dose forces to take into account the exposed number of individuals, which is a prerequisite in any radiological optimization.

This study was initiated by two observations. In nuclear and/or radiological emergency exercises decisions on countermeasures or protective actions are often mainly based on pre-defined reference levels and/or intervention levels. These intervention levels are in general based on individual (effective) dose levels and do not consider collective dose. In general, collective issues are only used in relation to the practical implementation of countermeasures (e.g. number of people to evacuate). The second observation is related to the difficulty to communicate or summarize differences in the impact of accidents/incidents with equal rating on the International Nuclear Event Scale (INES). Collective dose following nuclear and radiological accidents is evaluated by for instance the United Nations Scientific Committee on the Effects of Atomic Radiation (UNSCEAR, 2008). However, their approach is in general a post-accident analysis and consequently not useful for optimization in the preparedness and/or (early) response phase.

In this paper we investigate the use of collective dose in an emergency exposure situation based on three nuclear/radiological emergency exposure cases in the context of the above mentioned ICRP recommendations. The exposure scenarios include nuclear accidents as well as accidental radiological exposure situations of the public. To study the effect of the accumulation of low individual doses over extended time periods and for large geographical regions and/or large groups of people, the collective effective dose $(S)$ is studied as a function of a lower limit of the individual dose $\left(E_{1}\right)$ using the formula (ICRP, 2007):

$$
S\left(E_{1}, E_{2}, \Delta T\right)=\int_{E_{1}}^{E_{2}} E\left(\frac{d N}{d E}\right)_{\Delta T} d E,
$$

with $E$ the effective dose and $d N$ the number of individuals receiving a dose in the dose range $d E$ in the time period $\Delta T$. In this study the upper individual effective dose level $E_{2}$ corresponds always to the maximum calculated individual dose $\left(E_{2}=E_{\max }\right)$. Exposure of individuals leading to acute radiation effects is not excluded in the scenarios but is excluded in this study by applying some boundary conditions to the scenarios. The time period $\Delta T$ is always limited to a period of practical interest in emergency management. For $E_{1}$ the use of the ICRP (ICRP, 2007) effective dose reference levels for emergency exposure situations (20-100 $\mathrm{mSv}$ acute or per year) and the constraint of $1 \mathrm{mSv}$ for public exposure will be discussed.

\section{Case study}

All 3 cases studied are fictive (but realistic) nuclear or radiological accident scenarios. However, some simplifications had to be made compared to full reality to serve as exemplary scenarios. 
As a first case we consider a radiological accident caused by a lost sealed source of $100 \mathrm{TBq}$ of ${ }^{137} \mathrm{Cs}$ resulting in the external exposure to gamma radiation of a large group of people. Distance to source, shielding and exposure times can be very different for the people exposed resulting in a large variation in individual effective doses. To exclude acute effects we assume a minimum source distance of $5 \mathrm{~m}$ combined with a exposure time of $1 \mathrm{~h}$. To simplify - only for illustrative and calculation purposes - we assume a constant population density in the surrounding of the source of 1 person per square meter and an exposure of all individuals during 1 hour.

In this case the individual dose is only a function of distance (if we assume no shielding or only isotropic shielding) and the collective dose is calculated as a function of $E_{1}$ with no shielding (only attenuation of gamma radiation by air) and with limited shielding ( $1 \mathrm{~cm}$ of tissue equivalent shielding/10 meters distance, starting from $10 \mathrm{~m}$ onwards). The results are shown in Figure 1. Due to the attenuation of the radiation a limit (we will name it saturation value) of the collective dose is found if $E_{1}$ goes to zero. This saturation value of the collective dose mainly consists of contributions from individual doses larger than $1 \mathrm{mSv}$ (especially for the case with shielding which is most realistic).

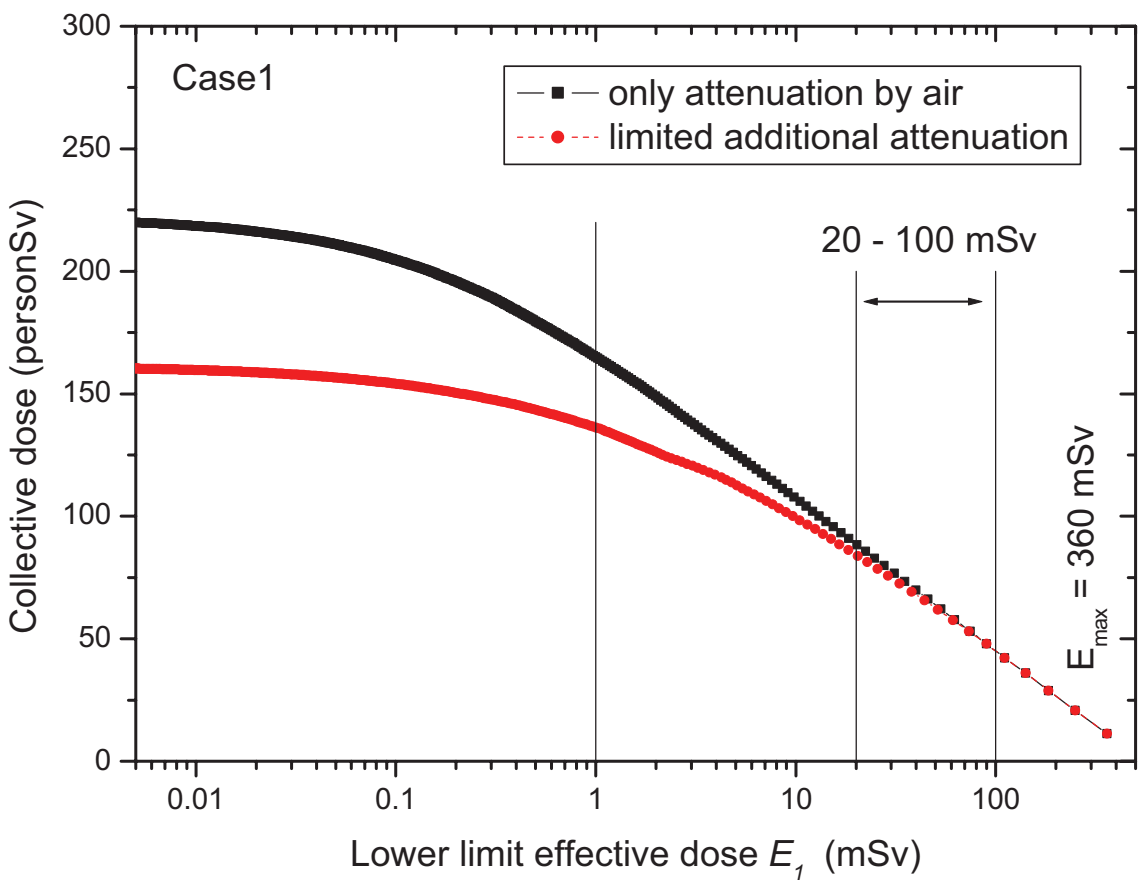

Figure 1 - Collective dose as a function of $E_{1}$ according to formula (1) for case 1 for a situation with only attenuation of the radiation by the air between the source and the exposed persons and for a limited additional attenuation.

The second scenario is the release of radioactive material into the atmosphere due to the explosion of a ${ }^{137} \mathrm{Cs}$ source of the same source strength as case 1 
(100 TBq). In this case the public in the environment is exposed to the dispersed radioactive material, in general via different pathways. Calculations are performed by using the Gaussian atmospheric dispersion model Hotspot v2.07.1 (Homann, 2010) with typical meteorological and dispersion parameters. We consider in this case only the effective dose from the exposure related to cloud passage (i.e. immersion and inhalation). We assume that the public is evacuated after plume passage and in this way not exposed to the ground shine from deposition or other pathways (ingestion...). Again a very high and uniform population density of 1 person per square meter is assumed. The collective dose as a function of the lower level is shown in Figure 2 for 3 different conditions. The first condition assumes that the plume is not depleted by dry deposition. Many atmospheric dispersion models (including HOTSPOT) ignore the depletion due to dry deposition because of the limited effect and the conservative character of this simplification. This oversimplification of physical reality - inconsistent with the conservation of dispersed mass - results in an ever increasing collective dose if the lower effective individual dose $\left(E_{1}\right)$ is lowered. If depletion is taking into account - corresponding to the depletion from wet deposition for two different rain intensities in the calculations shown in Figure 2 - the collective dose as a function of $E_{1}$ will reach a saturation value. Although this corresponds with the findings for case 1, also clear differences can be noted. In this case the saturation value of the collective dose (even with very large depletion factors due to heavy rain) can mainly be attributed to the accumulation of low or trivial individual doses for many persons.

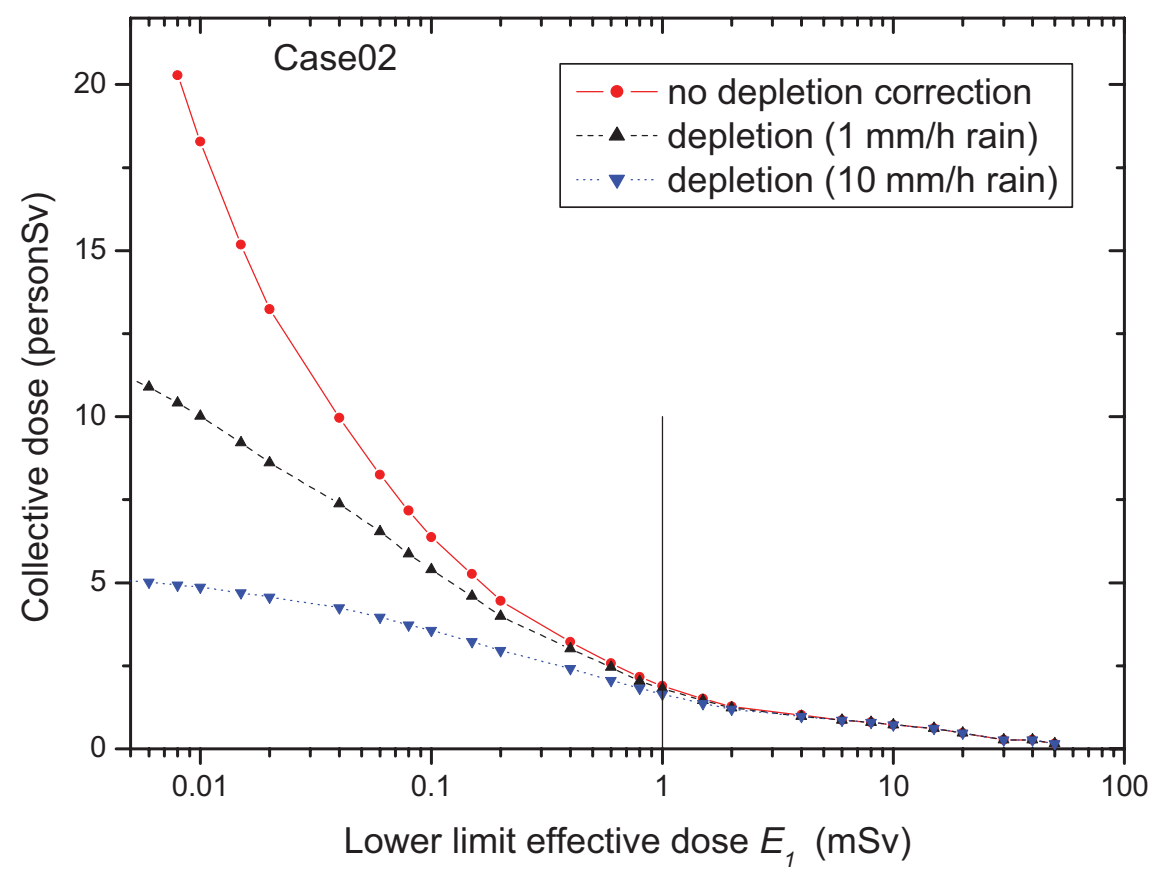

Figure 2 - Collective dose as a function of $E_{1}$ according to formula (1) for case 2 for a situation with no depletion of the plume and scenarios with depletion corresponding to moderate rain $(1 \mathrm{~mm} / \mathrm{h})$ and intense rain $(10 \mathrm{~mm} / \mathrm{h})$. 
This demonstrates that the evaluation of the collective dose as a function of $E_{1}$ can be used as an additional tool to study differences between scenarios. This can be of particular interest in the preparedness phase to get better insight in the collective impact of certain scenarios. Apart from the behaviour of collective dose as a function of $E_{1}$ the absolute value of collective dose for certain $E_{1}$ can be used to compare different accident scenarios.

In case 3 we consider the hypothetical exposure from ground shine in the aftermath of a large nuclear accident with a contamination of a large geographical region with ${ }^{137} \mathrm{Cs}$. The scenario is based on fictive accident calculations with the "NOODPLAN" model (Camps, 2010) around the Doel nuclear power plant located in the north of Belgium. ${ }^{137} \mathrm{Cs}$ deposition levels are in the order of magnitude
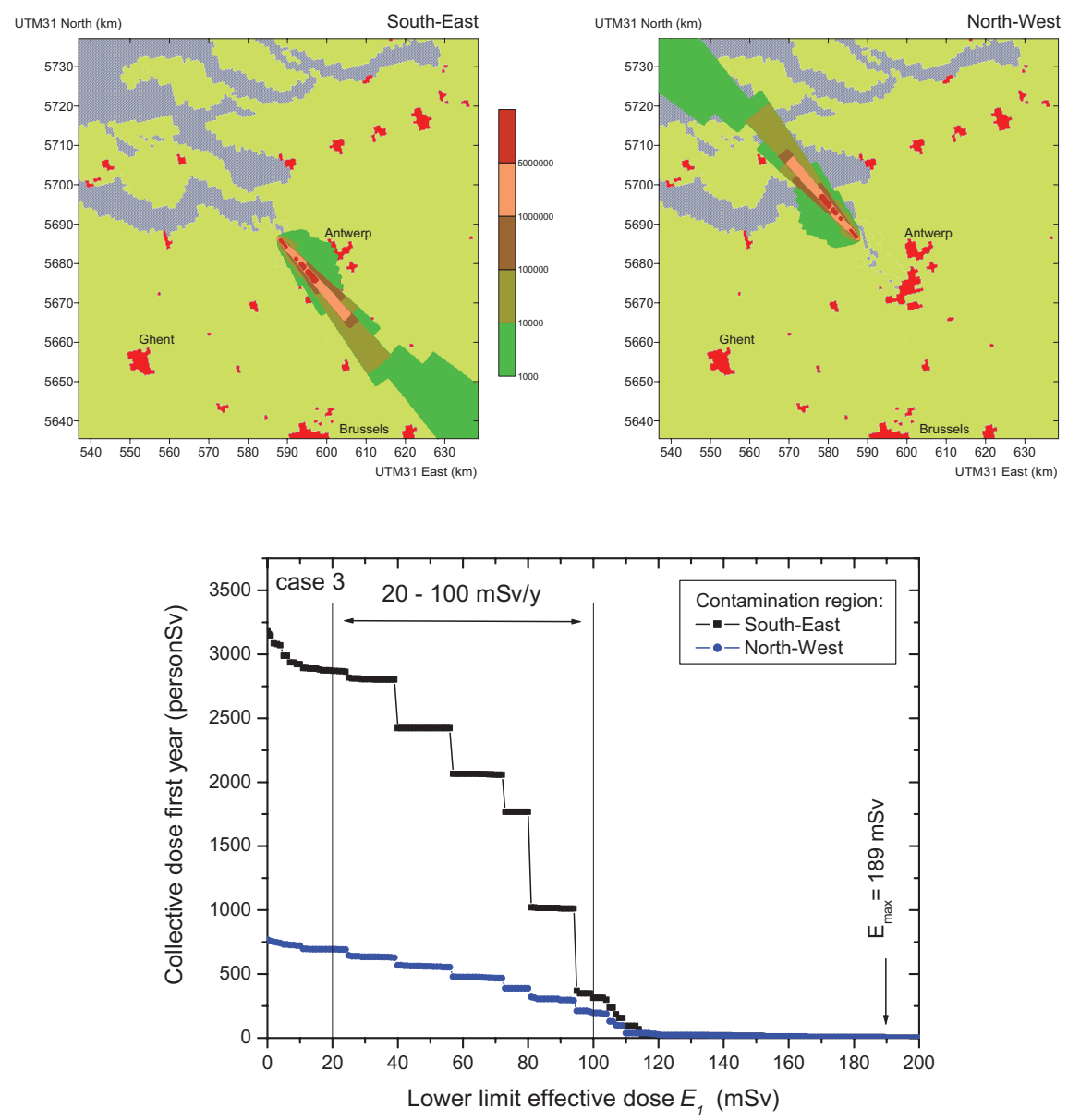

Figure 3 - Case 3. Upper left and upper right: the same contamination pattern (units $\mathrm{Bq} / \mathrm{m}^{2}$ ) on top of a simplified population density map (blue: water, green: average population density and red: urban environment) for two different affected regions (due to opposite wind direction). Bottom: collective dose from external radiation from the ${ }^{137} \mathrm{Cs}$ ground contamination as a function of $E_{1}$ according to formula (1) for the two affected regions shown in the upper maps. 
found after the Fukushima nuclear disaster in Japan. The collective dose is evaluated for an exposure time period $(\Delta T)$ of 1 year (first year). For this scenario the ICRP recommendations specifying the reference levels for individual effective doses are very clear. Further simplifications used in this scenario are the fact that the effect of leaching of the deposited radioactive material into the soil is not taken into account and that the population density is assumed to be constant for specific defined areas. Three areas are defined: water with zero population, average populated areas (372 people $/ \mathrm{km}^{2}$ which is more or less equivalent to the average population density in the region considered), and urban areas with a ten times higher population density ( 3720 people $/ \mathrm{km}^{2}$ ). The calculated deposition patterns and the collective dose calculations as a function of the lower individual effective dose are shown in Figure 3. Two sub scenarios are considered. One in which the accident affects a large neighbouring city (i.e. Antwerp) and another scenario with exact equal meteorological and dispersion parameters but with opposite wind directions (no large cities affected). The ground deposition patterns for the two sub scenarios and the collective dose corresponding to the exposure to ground shine for the first year as a function of the lower limit of the individual effective dose $E_{1}$ is shown in Figure 3. Similar to the fact that different meteorological conditions for the same accident scenario (same source term) can give large differences in individual effective dose values, this example shows clearly the effect on the collective dose when different geographical regions are affected with differences in population density. More interesting is the fact that actions implemented to reduce individual dose for people living in the regions exceeding the reference level of $100 \mathrm{mSv} / \mathrm{y}$ will have nearly no effect on the collective dose if this is calculated with $E_{1}$ equal to e.g. $20 \mathrm{mSv}$ (for the case in which the south-east region is affected). This is a clear example in which collective dose can be used in the context of optimization conform to the ICRP recommendations.

\section{Discussion}

Due to (the combination of) several physical factors such as shielding of radiation, depletion of the radioactive plume, radioactive decay, leaching, ... the collective dose as a function of the lower limit of individual doses will always reach a saturation value. The importance of trivial individual doses to the saturation value depends on the scenario. However for the cases in this study - which represent large nuclear accidents or radiological incidents with individual dose values exceeding the ICRP emergency reference levels - two out of the three cases can lead to an important fraction of the saturation collective dose from individual dose levels above $1 \mathrm{mSv}$ which corresponds to the dose constraint for the public in nonaccident conditions.

The calculation of the collective dose can have an important added value in the overall protection strategy avoiding following strictly pre-defined reference or intervention levels only defined on the basis of individual dose. The main advantage of using collective dose compared to e.g. evaluating the number of 
individuals exposed to a certain individual dose is the fact that collective dose integrates all this information and can give a point of reference for e.g. protective actions. A graphical representation of the collective dose as used in this study as a function of $E_{1}$ can be a useful tool. An alternative approach is the determination of the collective dose for specific values of $E_{1}$. The use of the values corresponding to ICRP reference levels and/or dose constraint are especially interesting if also the exposure time matches (respectively $20-100 \mathrm{mSv}$ acute or per year and $1 \mathrm{mSv} / \mathrm{y}$ ). Using collective dose in this way would give in addition to the INES scale a simple way of comparing accidents. In the aftermath of the Fukushima accident IRSN did a comparable analysis (IRSN, 2011). Apart from INES rating and collective dose specification also other factors such as the extent of contamination (surface) and the liquid releases should be included in an overall summary of an accident.

This study was limited to calculations of collective dose on the basis of models. The models should be of sufficient quality, cover the entire affected region and be of sufficient resolution. If measurements are available the collective dose calculations can be refined based on measured values.

To be of operational interest, as well for gaining experience during exercises as for real response, calculation of collective dose as specified by the ICRP recommendations and evaluated in this study should be introduced in a flexible way (user definition of $E_{1}, \Delta T, \ldots$ ) in decision support systems for nuclear and radiological emergencies.

\section{Conclusions}

Based on studying three nuclear/radiological accident scenarios it was found that the use of collective dose in combination with the (reference levels of the) individual dose as defined in the 2007 ICRP recommendations can be a useful tool in the preparedness as well as response phase of a nuclear/radiological accident. Especially plotting the collective dose as a function of the minimum individual effective dose or calculating the collective dose for specific values of minimum individual doses can give insight in collective exposure and can support decisions related to the overall protection strategy.

\section{REFERENCES}

Camps J. et al. (2010) The "NOODPLAN" early phase nuclear emergency models: an evaluation. In: Proceedings of the Third European IRPA Congress, 1418 June 2010, Helsinki, Finland.

Homann S.G. (2010) HOTSPOT version 2.07.1. National Atmospheric Release Advisory Center, Lawrence Livermore National Laboratory, https://narac. llnl.gov/HotSpot/HotSpot.html 
ICRP Publication 103 (2007) The 2007 Recommendations of the International Commission on Radiological Protection, Ann. ICRP 37 (2-4).

IRSN (2011)Assessment of the 66th day of projected external doses for populations living in the north-west fallout zone of the Fukushima nuclear accident, Institut de radioprotection et de sûreté nucléaire, report DRPH/2011-010.

UNSCEAR (2008) United Nations Scientific Committee on the Effects of Atomic Radiation 2008 report Vol. II - Effects of ionizing radiation, Annex C Radiation exposures in accidents. 